\title{
Inventário de ciclo de vida da manufatura de seringas odontológicas
}

\author{
Biagio F. GiannettI \\ Cecília M. Villas Bôas de Almeida \\ SiLVIA H. BonilLa \\ Celso Munhoz Ribeiro \\ UNIP
}

\begin{abstract}
Resumo
Uma das formas mais integradas, completas e eficazes para a gestão ambiental de atividades produtivas é baseada no ciclo de vida do produto, sendo a Avaliação do Ciclo de Vida sua principal ferramenta. O produto avaliado é a seringa odontológica tipo Carpule. O objetivo é a realização do Inventário de Ciclo de Vida (ICV) simplificado da fabricação de seringas odontológicas tipo Carpule, visando quantificar o uso de energia elétrica e a emissão de resíduos sólidos desde a mineração até o descarte do produto. A estrutura metodológica está baseada nas normas da série NBR ISO 14.040 e ISO 14.040. As fronteiras do estudo incluem as etapas de mineração, beneficiamento e manufatura; a produção do polietileno que compõe o tubete e a embalagem da agulha; a produção do aço, utilizado na fabricação da agulha, e o uso do conjunto pelos dentistas. A unidade funcional utilizada é $10^{6}$ (um milhão) de anestesias aplicadas. Na avaliação de melhorias, as soluções propostas permitem reduzir o consumo de energia elétrica em aproximadamente $20 \%$ na etapa de manufatura. A quantidade de resíduos sólidos pode ser reduzida de 40\% na etapa de manufatura, o que corresponde a aproximadamente 6,50\% do total de resíduos sólidos no ciclo de vida da seringa odontológica.
\end{abstract}

\section{Palavras-chave}

Inventário de ciclo de vida, seringa odontológica carpule, resíduos sólidos, consumo de energia elétrica, desmaterialização.

\section{Life cycle inventory of dental syringes}

\begin{abstract}
Among the methods proposed to environmental management of productive activities, Life Cycle Assessment is one of the most integrated, complete and efficacious. The product assessed is the dental carpule syringe. The aim of this work is to to perform a Streamlined Life Cycle Inventory (LCI) to quantify the electric energy consumption and the solid waste release from dental carpule syringes manufacture. The methodological structure is based on NBR ISO 14.040 and ISO 14.040 series. The LCl covers the syringe life cycle from mining to disposal. The limits of the system include the stages of copper and zinc mining and ore beneficiation; the production of polyethylene used for carpule and needle packing, the production of the steel for needles and syringe coils; and the use of the whole set by dentists. The functional unit was defined as $10^{6}$ (one million) applied anaesthesies. During improvement assessment, the proposed solutions allow reducing the electric energy consumption at about $20 \%$ in the manufacture stage. The solid waste quantity may be reduced nearly $40 \%$ in the manufacture stage, corresponding to approximately $6.50 \%$ of the total solid waste released during the whole life cycle of the dental carpule syringe.
\end{abstract}

Key words

Life cycle inventory, dental carpule syringe, solid wastes, electricity consumption, dematerialization. 


\section{INTRODUC̣̃̃O}

Atualmente, a avaliação ambiental é parte integrante das funções empresariais e pode ser realizada por meio de um Sistema de Gestão Ambiental (SGA), que é responsável pelas etapas de desenvolvimento, de implementação, de execução, de avaliação e de manutenção da política ambiental das empresas, segundo a Associação Brasileira de Normas Técnicas (ABNT, 1996). Esta avaliação ambiental tem por objetivo fundamentar e otimizar os processos decisórios que envolvem as atividades das empresas, melhorando o seu desempenho ambiental a partir da minimização do uso de recursos e do descarte de resíduos e da maximização de seus benefícios ambientais. Para tanto, as empresas buscam as ferramentas adequadas para avaliar seu desempenho ambiental. Uma delas, com foco no produto, é a Avaliação do Ciclo de Vida (ALTING; LEGARTH, 1995), que é a compilação e a avaliação das entradas, das saídas e dos impactos ambientais potenciais de um sistema de produto, ao longo de seu ciclo de vida (ABNT, 2001).

2001; NICOLETTI et al., 2002) e de diversos processos (SOMBEKKE et al., 1997; FINNVEDEN; EKVALL, 1998; FINNVEDEN, 1999; AZAPAGIC; CLIFT, 1999).

Devido à quantidade e à confiabilidade dos dados necessários para a análise e interpretação do impacto, a execução de uma Avaliação de Ciclo de Vida completa tem se mostrado não só custosa, mas também pouco prática. Um estudo de ACV requer a disponibilidade de grande quantidade de dados e bancos nacionais constituídos por inventários de ciclo de vida dos principais produtos e insumos utilizados pela sociedade. Desta forma, muitos pesquisadores e empresas têm optado pelo uso direto do ICV para a avaliação de sistemas produtivos, seja para propor melhorias (AHMADI et al., 2003; GEORGAKELLOS, 2005; SEYLER et al., 2005; RYDH; SUN, 2005), seja para contribuir para a consolidação de um banco de dados nacional (SILVA, KULAY, 2005; SILVA; BORGES, 2004; SILVA; KULAY, 2003). Atualmente, o ICV é também amplamente empregado diretamente como ferramenta para a gestão ambiental de grandes empresas (SEPPÄLA et al., 1998; VERSCHOOR; REIJNDERS, 1999; SEPPÄLA et al., 2002) para avaliar melhorias em produtos e processos com base nos resultados de uso de energia ou quantidade de emissões. Com o emprego dos resultados do inventário podem-se identificar possibilidades de melhoria e, principalmente, as relações do sistema em estudo com o meio ambiente (TILLMAN, 2000). Desta forma, é

A Avaliação de Ciclo de Vida (ACV) é uma ferramenta da gestão ambiental que identifica e avalia os impactos associados à existência de um produto. O ciclo de vida do produto tem início na extração dos recursos da natureza para a sua fabricação e termina quando o produto é descartado e retorna ao ambiente. Dentre as fases da $\mathrm{ACV}$, o inventário de ciclo de vida (ICV) é utilizado para avaliar os efeitos ambientais de um produto, processo ou atividade.

O ICV é amplamente empregado em todo o mundo há mais de quarenta anos (De SMET et al., 1996) e foi incorporado à ACV pela norma ISO 14041, que especifica os requisitos e os procedimentos necessários para a elaboração, interpretação e comunicação de um inventário de ciclo de vida. A fase de inventário de uma ACV corresponde à coleta e ao tratamento dos dados e informações, qualitativos e quantitativos, sobre os fluxos de materiais e energia envolvidos nas diversas interações com o meio ambiente que ocorrem no ciclo de vida de um produto ou serviço.

O ICV quantifica os recursos e as emissões desde a extração de matérias-primas até a sua disposição final e tem sido empregado como etapa da ACV na análise de diversos produtos (RIBEIRO et al., 2003; FURUHOLT, 1995; ANDERSSON et al., 1998; CEDERBERG; MATTSSON, 2000; BURGESS; BRENNAN, 2001; PAPASAVVA et al., possível modificar o sistema de forma direta com uma tomada de decisão ou indireta, influenciando o comportamento do mercado consumidor na busca de um produto com melhor desempenho ambiental.

Neste artigo, apresenta-se o ICV simplificado da fabricação de seringas odontológicas tipo Carpule visando quantificar o uso de energia elétrica e a emissão de resíduos sólidos desde a mineração até o descarte do produto. Vale ressaltar que este estudo, ao contrário dos encontrados na literatura, trata de uma empresa de pequeno porte.

\section{METODOLOGIA}

Normas nacionais e internacionais especificam os requisitos e os procedimentos necessários para a elaboração, interpretação e comunicação de um Inventário de Ciclo de Vida, como parte da Avaliação de Ciclo de Vida. O estudo da ACV segue a estrutura metodológica proposta pela ISO e pela ABNT, dividindo-se as normas para a realização de cada fase (Tabela 1).

Os resultados são estruturados de acordo com as normas e tal estrutura determina as fases e os procedimentos gerais da execução do estudo de ACV, de acordo com as normas mundiais e as correspondentes nacionais. As características- 
chave para a realização de um estudo de $\mathrm{ACV}$, segundo a ABNT (2001), são:

- abordagem sistemática e adequada com relação aos aspectos ambientais de sistemas de produto, desde a aquisição de matéria-prima até a disposição final;

- possibilidade de variação do detalhe e do período de tempo de um estudo da ACV, dependendo da definição do objetivo e do escopo;

- transparência quanto ao escopo, suposições, descrição da qualidade dos dados, dos métodos e apresentação dos resultados;

- possibilidade de inclusão de novas descobertas científicas e melhoria no estado da arte da tecnologia;

- inexistência de base científica para reduzir resultados da $\mathrm{ACV}$ a um único número ou pontuação globais;
- inexistência de um único método para conduzir estudos da ACV.

A Figura 1 mostra as fases de uma Avaliação de Ciclo de Vida, em que se destacam as etapas utilizadas neste estudo. A avaliação de melhorias é subseqüente à realização do inventário, sendo que a avaliação do impacto não foi realizada.

Na seleção do escopo da análise, as fronteiras do sistema são definidas e determina-se o objetivo da análise. $\mathrm{O}$ objetivo deste estudo é a realização do inventário do ciclo de vida da seringa para injeção odontológica metálica produzida e consumida no Brasil. O escopo do estudo é o de identificar as etapas em que ocorrem o maior consumo de energia elétrica e o maior descarte de resíduos sólidos

Tabela 1: Fases da ACV e as respectivas normas nacionais e internacionais que regulamentam cada fase.

\begin{tabular}{|l|l|l|}
\hline \multicolumn{1}{|c|}{ FASES DA ACV } & \multicolumn{1}{|c|}{ NORMAS } \\
\hline 1. & Definição do objetivo e escopo & ISO 14040, ISO 14041, NBR ISO 14040, NBR ISO 14041 \\
\hline 2. & Análise do inventário do ciclo de vida & ISO 14040, ISO 14041, NBR ISO 14040, NBR ISO 14041 \\
\hline 3. & Avaliação do impacto do ciclo de vida & ISO 14040, ISO 14042, NBR ISO 14040, NBR ISO 14042 \\
\hline 4. & Interpretação & ISO 14040, ISO 14043, NBR ISO 14040 \\
\hline
\end{tabular}

Figura 1: Fases da Avaliação de Ciclo de Vida e os objetivos deste estudo.

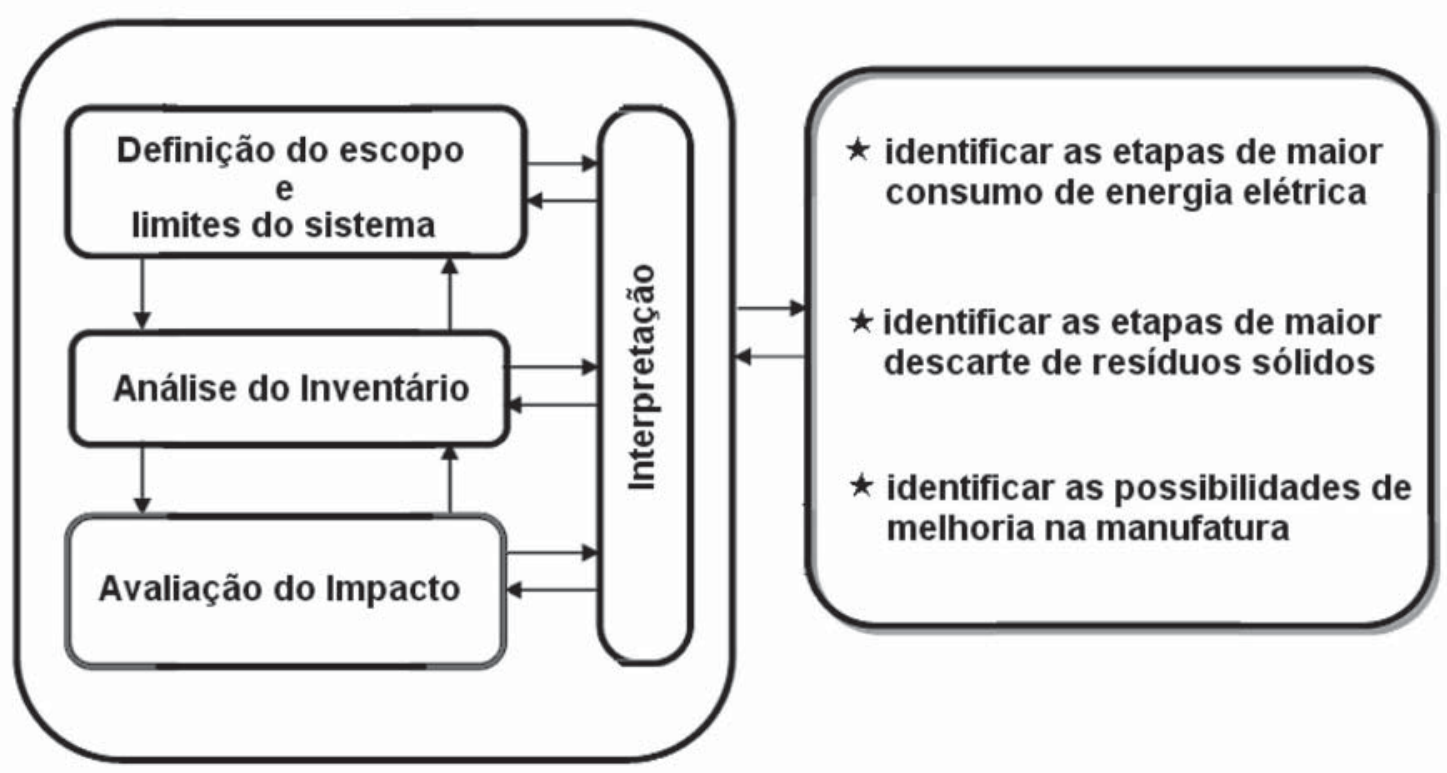


decorrentes da produção de uma seringa para injeção odontológica tipo Carpule e as possibilidades de melhoria na fase de manufatura.

Como o ICV visa quantificar os materiais e a energia que cruzam as fronteiras de um sistema, a determinação dos limites do sistema é uma etapa crítica, pois se baseia em uma variedade de fatores que dependem, principalmente, do objetivo da análise e da disponibilidade de dados confiáveis. O resultado do inventário é uma listagem de materiais e da energia requerida, produtos, co-produtos e resíduos que são descartados. Esta listagem pode ser chamada de balanço de material e energia, tabela de inventário ou eco-balanço do produto. Com base nos resultados do inventário, as possibilidades de melhoria do sistema são exploradas.

A elaboração do inventário de ciclo de vida para as seringas odontológicas tipo Carpule foi dividida nas etapas mostradas na Tabela 2.

\section{Descrição do sistema e estabelecimento dos limites do sistema}

O ponto de partida do sistema em estudo é uma indústria de pequeno porte, fundada em 1951, localizada em São Paulo, fabricante de aparelhos odontológicos. O ICV focaliza a seringa odontológica de latão tipo Carpule utilizada para a aplicação de anestesias (Fig. 2). Na Figura 2, a seta correspondente à bucha é tracejada, pois esta peça encontra-se no interior da montagem, não sendo visualizada na figura. Na montagem da seringa utiliza-se ainda uma mola de aço, comprada de terceiros.

As seringas tipo Carpule são metálicas e fabricadas em latão com revestimento de cromo, possuem tamanho padrão, podendo ter ou não dispositivo para refluxo (permite a aspiração para o tubete de conteúdo sangüíneo, constituindo um instrumento de prevenção de injeção indevida de anestésico no meio intravascular). São utilizadas principalmente para a injeção do anestésico. A agulha é composta de uma ponta de

Tabela 2: Etapas para a realização do ICV utilizadas neste estudo.

\begin{tabular}{|l|c|}
\hline \multicolumn{1}{|c|}{ ETAPA } & ITEM \\
\hline Descrição do sistema e estabelecimento dos limites do sistema & 2.1 \\
\hline Identificação dos resíduos e do consumo de energia elétrica por etapa do ciclo de vida da seringa & 2.2 \\
\hline Coleta de dados primários & 2.2.4. e 2.2.5 \\
\hline Coleta de dados secundários & 2.2.1. a 2.2.3 \\
\hline Definição da Unidade Funcional & 2.3 \\
\hline
\end{tabular}

Figura 2: Seringa odontológica para anestesia tipo Carpule, com a nomenclatura utilizada para cada peça pelo fabricante.

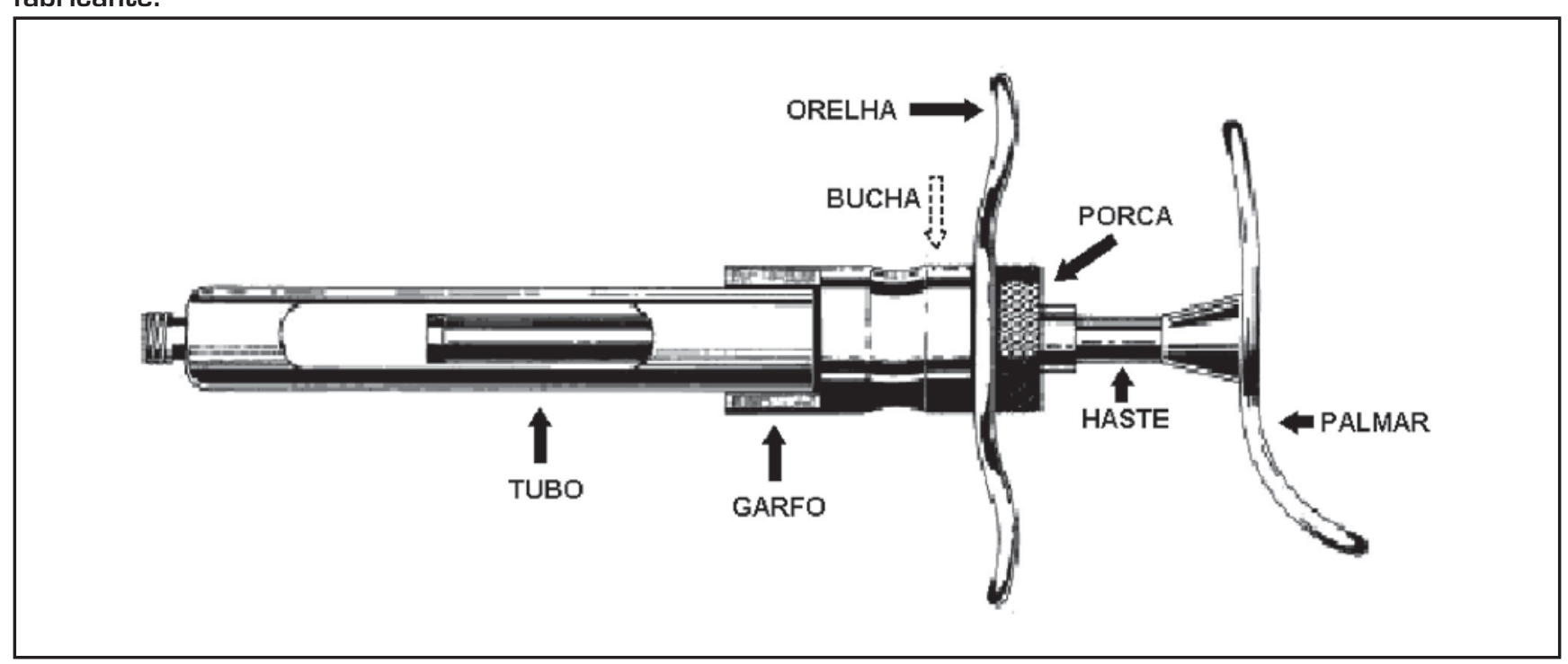


aço, cortada em bisel para facilitar a penetração nos tecidos e direcionar convenientemente o jato do líquido injetado, e uma parte de plástico que permite o rosqueamento no tubo da seringa Carpule (Fig. 3a). É fornecida em uma embalagem de plástico que protege a ponta. Os tubetes são tubos plásticos que contêm a solução anestésica, têm uma extremidade metálica e uma extremidade de borracha (Fig. 3b). Para inserir o tubete de anestésico na Carpule, é necessário tracionar a haste-êmbolo da mesma exibindo o receptáculo do tubete, que deve ser inserido com o anel metálico voltado para o local onde será atarraxada a agulha.

As fronteiras definidas para o sistema incluem as etapas de (i) mineração, beneficiamento e manufatura das partes de latão; (ii) a produção do polietileno que compõe o tubete, parte da agulha e a embalagem da agulha; (iii) a produção do aço, utilizado na fabricação da agulha e da mola empregada na seringa e (iv) o uso do conjunto (seringa, agulha e tubete de anestésico) pelos dentistas (Fig. 4). O sistema foi avaliado no que refere ao consumo de energia elétrica e ao descarte de resíduos sólidos em todas as etapas. O transporte entre as etapas não foi considerado.
Observa-se na Figura 4 que os resíduos sólidos que resultam da cadeia produtiva da seringa odontológica são provenientes das etapas de extração e do beneficiamento do latão, da produção de aço e polietileno e da etapa de uso da seringa, quando são descartados tubetes e agulhas descartáveis. Na etapa de manufatura, os resíduos de metal são vendidos a sucateiros. O resíduo sólido gerado no tratamento da superfície da seringa, que é terceirizado pela manufatura (lodo galvânico, não mostrado na figura), também foi considerado na tabela do inventário.

A reciclagem foi representada com linhas pontilhadas, pois não é exclusiva do sistema em estudo, mas é propriedade de um sistema maior, que inclui todas as outras empresas que utilizam o latão, o aço, o plástico e o papel como matériaprima. Neste sistema maior, algumas empresas reciclam internamente parte do material que utilizam, mas a maioria delas, como a manufatura de seringas odontológicas, vende o material não utilizado para sucateiros, o que torna possível a circulação de material e aumenta a eficiência de seu uso. Os fluxos associados à reciclagem não foram contabilizados, pois estão fora dos limites estabelecidos neste estudo.

Figura 3: Ilustração da agulha para anestesia (a) e do tubete anestésico (b).

(a)

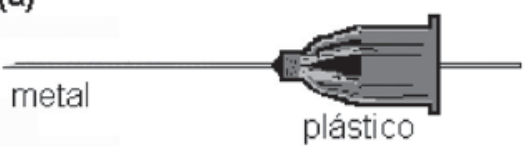

(b)

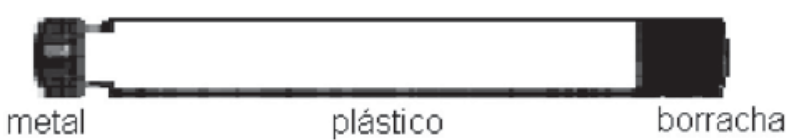

Figura 4: Limites estabelecidos para o sistema da fabricação de seringas odontológicas. Os fluxos mostrados referem-se aos resíduos sólidos; [*] metal e [**] embalagens.

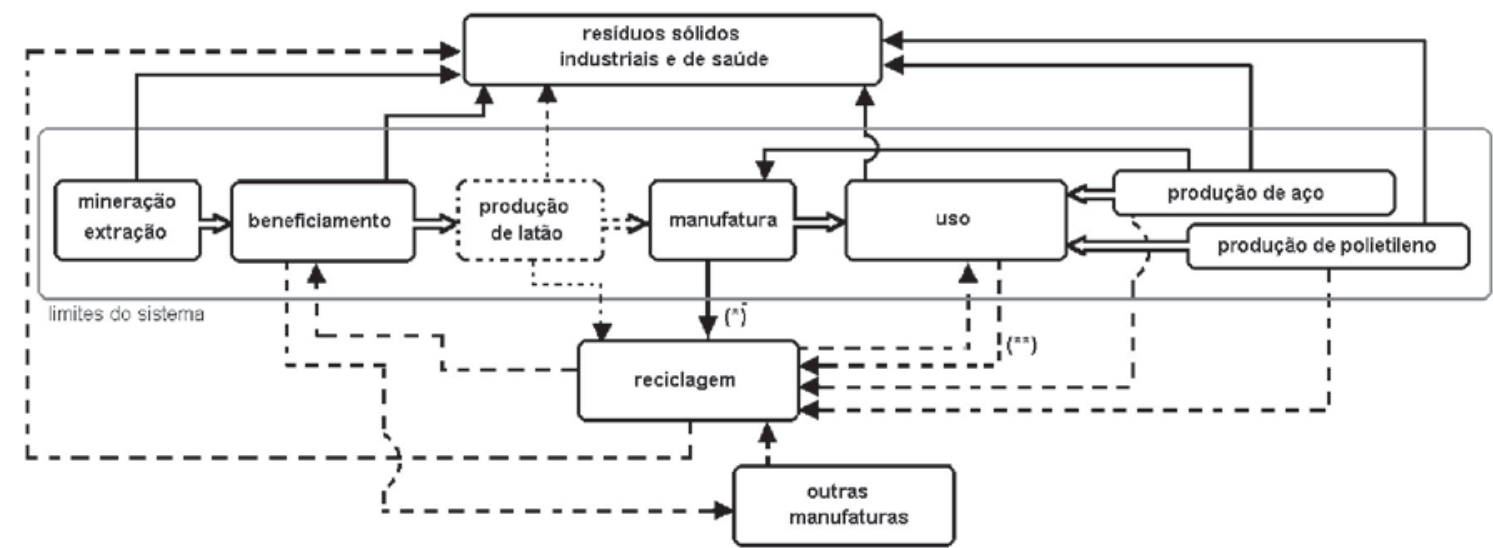




\section{Identificação dos resíduos e do consumo de energia elétrica por etapa do ciclo de vida}

\section{Mineração e beneficiamento}

A seringa odontológica para anestesia é fabricada com latão, o que remete à mineração e ao beneficiamento do cobre e do zinco. Foram utilizados dados da produção média mundial dos dois metais para o cálculo da quantidade dos resíduos sólidos e da energia consumida nestas duas etapas (AYRES et al., 2000). A produção mundial de cobre constitui-se de $73 \%$ de cobre obtido de minérios sulfetados e $27 \%$ de minérios contendo óxido de cobre, em massa. Cada tipo de minério gera diferentes tipos de resíduos, tanto em quantidade como em qualidade (Tabela 3). A porcentagem média em massa de cobre é de $0,65 \%$ nos minérios sulfetados e de $0,58 \%$ nos minérios que contém óxido de cobre. O zinco também é obtido de minérios sulfetados $(75 \%)$ e de minério contendo óxido de zinco $(25 \%)$ e os resíduos gerados também dependem do tipo de minério do qual o metal é extraído (Tabela 3). $\mathrm{O}$ conteúdo de zinco é de 8,3\% nos minérios de óxido de zinco e de $9 \%$ nos minérios sulfetados. Os valores mostrados na Tabela 3 referem-se à obtenção de um quilograma de cada um dos metais.

O latão para a fabricação da seringa odontológica é constituído de $65 \%$ de cobre e $35 \%$ de zinco, o que gera aproximadamente $130.000 \mathrm{~kg}$ de resíduos na mineração e 4.500 $\mathrm{kg}$ de resíduos no beneficiamento para a produção de cada tonelada de latão utilizado. Os resíduos da produção de latão (mostrada em linhas pontilhadas na Figura 4) a partir dos dois metais não foram considerados neste trabalho devido à ausência de dados disponíveis.

Um estudo do padrão mundial do consumo de energia

Tabela 3: Quantidade de resíduos sólidos na mineração e no beneficiamento do cobre e do zinco (média mundial).

\begin{tabular}{|c|c|c|}
\hline & $\mathrm{kg}_{\text {REsiduo }} / \mathrm{kg}_{\text {MEtAL }}$ & \\
\hline $\begin{array}{l}\text { Resíduos na mineração para cobre } \\
\text { Resíduos sólidos da extração a partir do óxido de cobre } \\
\text { Resíduos sólidos da extração a partir do sulfeto de cobre } \\
\text { Subtotal }\end{array}$ & $\begin{array}{r}47,45 \\
141,65\end{array}$ & 189,10 \\
\hline $\begin{array}{l}\text { Resíduos no beneficiamento para cobre } \\
\text { Sólidos da lixiviação } \\
\text { Escória } \\
\text { Lodo } \\
\text { Subtotal }\end{array}$ & $\begin{array}{l}3,14 \\
2,95 \\
0,03\end{array}$ & 3,23 \\
\hline $\begin{array}{l}\text { Resíduos na mineração para zinco } \\
\text { Resíduos sólidos da extração a partir do óxido de zinco } \\
\text { Resíduos sólidos da extração a partir do sulfeto de zinco } \\
\text { Subtotal }\end{array}$ & $\begin{array}{r}2,90 \\
12,45\end{array}$ & 15,35 \\
\hline $\begin{array}{l}\text { Resíduos no beneficiamento para zinco } \\
\text { Partículas de poeira } \\
\text { Escória } \\
\text { Lodo } \\
\text { Subtotal }\end{array}$ & $\begin{array}{l}0,04 \\
1,02 \\
0,39 \\
\text { Total }\end{array}$ & $\begin{array}{r}1,45 \\
\mathbf{2 0 9 , 1 3}\end{array}$ \\
\hline
\end{tabular}

Fonte: Ayres et al., 2000. 
elétrica para a produção de zinco mostrou que são necessários aproximadamente $17.000 \mathrm{kWh}$ para a extração e $20.000 \mathrm{kWh}$ para o beneficiamento de uma tonelada de zinco (Report of Energy and Environmental Profile of the U.S. Mining Industry, 2001a). No caso do cobre, utiliza-se $35.000 \mathrm{kWh}$ e $21.000 \mathrm{kWh}$ na mineração e no beneficiamento de uma tonelada do metal, respectivamente (Report of Energy and Environmental Profile of the U.S. Mining Industry , 2001b).

\section{Fabricação do aço}

Os dados foram retirados do estudo mundial de um inventário de ciclo de vida para os produtos de aço, realizado em 1996 pelo Instituto Internacional de Ferro e Aço (IISI) e atualizado nos anos de 1999 e 2000 . O inventário foi divulgado com o objetivo de coletar dados necessários para uma Análise do Ciclo de Vida, estimular a otimização do desempenho ambiental dos processos de produção do aço e promover iniciativas para aumentar a reutilização do aço. A quantidade de resíduos sólidos para a produção de $1 \mathrm{~kg}$ de aço é de 1,75 kg com um consumo de 111,24 kWh (IISI, 2000). A Tabela 4 mostra, resumidamente, as quantidades de resíduos sólidos relativas à produção de $1 \mathrm{~kg}$ de aço.

\section{Fabricação do polietileno}

Os dados foram obtidos no relatório publicado pela associação das indústrias de plástico européias em 2003. Os dados que constam do eco-perfil publicado foram obtidos de 27 plantas que produzem 4,48 milhões de toneladas de polietileno e representam $93,5 \%$ da produção européia. $\mathrm{O}$ consumo médio de energia para produzir $1 \mathrm{~kg}$ de polietileno equivale a $280,80 \mathrm{kWh}$, variando entre $230,40 \mathrm{kWh}$ e 345,60 $\mathrm{kWh}$. A quantidade de resíduos sólidos é de $0,041 \mathrm{~kg}$ por quilograma de polietileno produzido (BOUSTEAD, 2003).

\section{Manufatura}

Os dados desta etapa foram obtidos diretamente da linha de produção da empresa de pequeno porte fabricante da seringa odontológica.

No ano de 2002 foram produzidas 5.020 seringas para anestesia, que são formadas por sete peças de latão distintas. Para cada uma das sete peças que compõem a seringa para injeção odontológica (Fig. 2), obtiveram-se as massas de resíduos sólidos geradas na usinagem do latão (Tabela 5).

O consumo de energia elétrica necessário para a produção das 5.020 seringas no ano de 2002 foi de $582,47 \mathrm{kWh}$. Este valor foi obtido a partir da medição do tempo de usinagem

Tabela 4: Quantidade de resíduos sólidos na produção de aço na Europa.

\begin{tabular}{|c|c|}
\hline & $\mathbf{k g} / \mathbf{k g}$ \\
\hline Sucata & 0,14 \\
\hline Lodo & 1,43 \\
\hline Total & 0,18 \\
\hline
\end{tabular}

Fonte: IISI, 2000.

Tabela 5: Massas de resíduos sólidos das peças componentes da seringa odontológica, 2002.

\begin{tabular}{|c|c|}
\hline PEÇA & RESÍDuO SÓLIDO $\mathbf{k g} / \mathbf{A N O}$ \\
\hline Tubo & 256,02 \\
Haste & 10,04 \\
Garfo & 200,80 \\
Bucha & 25,10 \\
Porca & 55,22 \\
Orelha & 80,32 \\
Palmar & 65,26 \\
\hline Resíduo Sólido Total & $\mathbf{6 9 2 , 7 6}$ \\
\hline
\end{tabular}


e multiplicado pela potência do motor do torno e da prensa utilizados em cada processo.

$\mathrm{O}$ tratamento de superfícies é terceirizado e equivale a $0,2 \%$ do consumo total do fornecedor, que é de $66.000 \mathrm{kWh}$ por ano. A quantidade de resíduo sólido (lodo galvânico) gerada pelo tratamento de superfície da seringa odontológica foi estimada em $0,83 \mathrm{~kg}$ por ano.

A montagem do produto é realizada em um ambiente alimentado por quatro lâmpadas de 40W. Montam-se 100 (cem) seringas para injeção em 2 (duas) horas, sendo o consumo anual de energia elétrica de $16,06 \mathrm{kWh}$ nesta etapa. A embalagem do produto é realizada em um ambiente alimentado por quatro lâmpadas de 40W. Embalam-se 200 (duzentas) seringas para injeção em 1 (uma) hora, com um consumo anual de 4,02 kWh. A embalagem é constituída de uma caixa de papelão com massa de $0,009 \mathrm{~kg}$ (papelão $0,300 \mathrm{~kg} / \mathrm{m}^{2}$ ) e um saco plástico polipropileno com massa de $0,00083 \mathrm{~kg} /$ unidade. A expedição do produto é feita por caminhões de redes distribuidoras, em todo o território brasileiro mas, como citado anteriormente, o transporte não é objeto do presente estudo.

igual número de agulhas descartáveis $(0,0002 \mathrm{~kg} /$ unidade $)$ e de embalagens para agulha $(0,0016 \mathrm{~kg} /$ unidade $)$, de acordo com os valores obtidos por meio do questionário.

\section{Definição da unidade funcional}

A unidade funcional (UF), claramente definida, mensurável e relevante em relação aos elementos do sistema, é a base que permite comparações entre estudos de ciclo de vida, avaliação ou inventário. Ao definir a unidade funcional, deve-se considerar não a quantidade de produto, mas a função que este desempenha. Para uma mesma função, pode haver vários produtos, constituídos de diferentes materiais, em quantidade e qualidade. Desta forma, a unidade funcional deve se referir à função a que este produto se destina. No caso das seringas odontológicas, a função é a aplicação de anestesias e a unidade funcional utilizada é $10^{6}$ (um milhão) de anestesias aplicadas. Para o cálculo da quantidade de energia requerida e da quantidade de resíduos sólidos de cada processo considerou-se o número de dentistas atuantes no Brasil em 2002, 197.022 dentistas (CFO, 2003), o número de anestesias aplicadas neste mesmo ano (173.379.360 anestesias), o número de seringas por dentista (6 seringas por dentista) e a vida útil de cada seringa (20 anos). energia elétrica e emissão de resíduos sólidos desde a mineração até o descarte.

\section{RESULTADOS}

\section{Inventário de ciclo de vida}

Uso

Os dados deste estágio foram obtidos por meio de questionários respondidos por dentistas da cidade de São Paulo em março de 2003. Foram entrevistados cinqüenta profissionais distribuídos da seguinte forma: dez clínicos gerais, oito especialistas em cirurgia bucomaxilofacial, oito em endodontia, oito em odontopediatria, oito em patologia bucal e oito em periodontia. Todos os cinqüenta entrevistados exercem a profissão há pelo menos 25 anos. Com os resultados obtidos do questionário, foi possível determinar quantas seringas cada dentista possui, o tempo de vida médio das seringas, quantas anestesias são aplicadas por dia, qual a freqüência das esterilizações e o consumo de energia elétrica das autoclaves.

A utilização do produto pelo profissional de odontologia envolve ainda o descarte das seguintes partes: uma caixa de papelão por seringa, um saco plástico por seringa, um tubete de anestésico por anestesia aplicada e uma agulha descartável por anestesia aplicada. Para o ano de 2002, a compra de 5.020 seringas pelos dentistas acarretou um descarte de 5.020 caixas de papelão e 5.020 sacos plásticos de polipropileno. Por outro lado, houve o descarte de 173.379.360 tubetes de anestésico (0,0017 kg/unidade) e
Os resultados obtidos permitem a construção de tabelas de inventário que mostram a geração de resíduos sólidos (Tabela 6) em todos os sete estágios de ciclo de vida da seringa para injeção odontológica.

Deve-se destacar que os dados relativos ao descarte do tubete de anestésico levam em consideração sua massa total que, na verdade, é constituída de três materiais: polietileno, borracha e alumínio. A massa de borracha e alumínio foi considerada desprezível em relação à do plástico. No item do descarte da agulha para injeção, foram contabilizadas as massas de aço inoxidável e polietileno.

$\mathrm{Na}$ coluna "Impacto Potencial" são listados os possíveis impactos causados pelo descarte dos materiais ao longo do ciclo de vida da seringa odontológica. Cabe lembrar que toxicidade humana refere-se à toxicidade para humanos causada pela dispersão do material no ambiente, enquanto que ecotoxicidade refere-se à toxicidade em ecossistemas aquáticos, causada pela dispersão de material descartado no ambiente, como por exemplo, a dispersão de metais pesados provenientes do lodo descartado no beneficiamento do cobre e do zinco (ISO/TR 14047, 2003).

O consumo de energia elétrica (Tabela 7) registrado ao 
Tabela 6: Resíduos sólidos gerados em cada etapa do ciclo de vida da seringa para injeção odontológica para $10^{6}$ anestesias aplicadas (UF).

\begin{tabular}{|c|c|c|}
\hline ETAPA & $\begin{array}{c}\text { RESÍDUO SÓLIDO } \\
\text { kg / UF }\end{array}$ & PRINGIPAIS IMPAGTOS POTENCIAIS \\
\hline $\begin{array}{l}\text { Mineração } \\
\qquad \begin{array}{r}\text { Extração (latão) } \\
\text { cobre } \\
\text { zinco } \\
\text { beneficiamento (latão) } \\
\text { cobre } \\
\text { zinco }\end{array}\end{array}$ & $\begin{array}{l}588,22 \\
316,73 \\
190,16 \\
102,39 \\
1197,50\end{array}$ & $\begin{array}{l}\text { Exaustão de recursos não renováveis e } \\
\text { Ecotoxicidade }\end{array}$ \\
\hline Fabricação do aço & 54,19 & Exaustão de recursos não renováveis \\
\hline Fabricação do polietileno & 140,35 & Exaustão de recursos não renováveis \\
\hline $\begin{array}{l}\text { Manufatura } \\
\text { usinagem } \\
\text { mola } \\
\text { aço } \\
\text { tratamento de superfícies } \\
\text { subtotal }\end{array}$ & $\begin{array}{l}47,05 \\
0,92 \\
<0,01 \\
\mathbf{4 7 , 9 7}\end{array}$ & Toxicidade humana, ecotoxicidade e acidificação \\
\hline $\begin{array}{l}\text { Uso } \\
\text { polietileno } \\
\text { aço } \\
\text { embalha polietileno } \\
\text { polietileno } \\
\text { saco plástico } \\
\text { polietileno } \\
\text { caixa de papelão }\end{array}$ & $\begin{array}{c}180,00 \\
30,00 \\
1620,00 \\
1640,00 \\
<0,01 \\
<0,01 \\
\mathbf{3 5 7 0 , 0 0}\end{array}$ & Toxicidade humana \\
\hline Total & 5010,01 & \\
\hline
\end{tabular}


longo do ciclo de vida da seringa odontológica mostra que, apesar do alto consumo de energia nas etapas de extração e beneficiamento, o maior consumo ocorre na etapa de uso das seringas, que são esterilizadas, em média, duas vezes ao dia em autoclave. nos processos de manufatura exercem sobre o ciclo de vida total é muitas vezes desprezada ou negligenciada. Por outro lado, observou-se que uma avaliação de melhorias com base no ICV pode trazer benefícios tanto na manufatura como nas demais etapas do ciclo de vida de um produto. As melhorias propostas baseiam-se na desmaterialização do processo de manufatura. A desmaterialização é uma das ferramentas da Ecologia Industrial que remete à diminuição do uso de matériasprimas nos processos.

\section{Resíduos sólidos}

Apesar de os resíduos sólidos da fase de ma-

\section{Avaliação de melhorias}

Uma das vantagens de avaliar o ciclo de vida de um produto é conhecer detalhadamente cada etapa necessária para sua produção e utilização. Entretanto, esta informação não permite à manufatura qualquer interferência nas etapas anteriores ou posteriores. Este fato se agrava quando se trata de uma empresa de pequeno porte como a avaliada neste estudo. Uma empresa deste tipo não pode influenciar os processos da mineração, nem o projeto do produto, já que contribui com uma pequena parcela do mercado. Com base neste tipo de argumento, a influência que mudanças, mesmo pequenas, nufatura representarem aproximadamente $0,3 \%$ em massa dos resíduos totais gerados pela produção da seringa odontológica ao longo de seu ciclo de vida, a elaboração do ICV permitiu identificar possibilidades de melhorias nesta etapa. Foi observado que, para cada seringa de massa $0,08 \mathrm{~kg}$, são gerados aproximadamente $0,14 \mathrm{~kg}$ de resíduos de latão, ou seja, $37 \%$ do material que entra na empresa permanecem na seringa e os restantes $63 \%$ passam pela empresa e são vendidos como sucata. A maior quantidade de resíduos foi verificada no processo de usinagem das peças tubo e garfo (Tabela 4). Neste processo, o tubo da seringa é feito a partir

Tabela 7: Consumo de energia elétrica em cada estágio do ciclo de vida da seringa para injeção odontológica para $10^{6}$ anestesias aplicadas (UF).

\begin{tabular}{|c|c|c|}
\hline & & kWh / UF \\
\hline \multicolumn{3}{|l|}{ Mineração } \\
\hline extração & \multirow{4}{*}{ subtotal } & $1.962,89$ \\
\hline beneficiamento & & $1.390,84$ \\
\hline & & $3.353,73$ \\
\hline Fabricação do aço & & 955,43 \\
\hline Fabricação do polietileno & & $10.947,30$ \\
\hline \multicolumn{3}{|l|}{ Manufatura } \\
\hline usinagem & & 39,56 \\
\hline tratamento de superfície & & 8,96 \\
\hline montagem & & 1,09 \\
\hline embalagem & & 0,27 \\
\hline subtotal & & 49,88 \\
\hline \multicolumn{2}{|l|}{ Uso } & $37.500,00$ \\
\hline & Total & $52.806,04$ \\
\hline
\end{tabular}


de um vergalhão redondo, do qual o latão é retirado para acomodar os itens que compõem o interior da seringa (haste, bucha e tubete). A massa de resíduo sólido $(0,051 \mathrm{~kg})$ neste caso corresponde a aproximadamente 3,5 vezes a do produto $(0,015 \mathrm{~kg})$. No caso do garfo, também usinado, a relação entre resíduo sólido e peça acabada é de 3:1 em massa. A solução proposta foi a substituição dos vergalhões redondos de latão por tubos com dimensões condizentes.

Com a solução encontrada, a geração anual de resíduo sólido na manufatura do tubo e do garfo seria reduzida de $256,02 \mathrm{~kg}$ para $43,52 \mathrm{~kg}$ e de $200,80 \mathrm{~kg}$ para $142,57 \mathrm{~kg}$, respectivamente, com uma redução total de aproximadamente $40 \%$. Pode-se, também, afirmar que ao diminuir a quantidade de latão utilizada no processo de usinagem, pela substituição de vergalhões por tubos, ocorre proporcional diminuição nas quantidades necessárias de cobre e zinco extraídas, o que reduz a exaustão de recursos não renováveis. Com a mudança no processo de manufatura do tubo e do garfo, a massa de material utilizada na fabricação da seringa odontológica seria reduzida em aproximadamente $25 \%$. A Figura 5 mostra uma estimativa dos benefícios considerando uma redução proporcional dos resíduos sólidos nas etapas de mineração e beneficiamento.

$\mathrm{Na}$ etapa do tratamento de superfície, apesar da quantidade de resíduos relativamente pequena $(0,06 \%)$, deve-se levar em conta em uma futura avaliação que o lodo químico é depositado em aterros e pode causar impactos de toxicidade humana pela exposição a substâncias tóxicas, de ecotoxicidade com danos causados à flora e à fauna e acidificação.

$\mathrm{O}$ destino da caixa de papelão e do saco plástico que servem de embalagem para o produto foram considerados de responsabilidade do fabricante e a melhor solução encontrada foi a substituição destes dois itens por uma embalagem que consiste em um saco de papel com janela de papel celofane, que continua sendo reciclável. Neste caso, além da diminuição da massa de resíduos descartada da etapa de uso do produto, houve ainda uma redução de dez vezes no custo da embalagem.

Os resíduos sólidos na fase de uso representam 74,2\% do total, sendo 4,4\% relativos ao descarte das agulhas, 33,6\% ao de sua embalagem plástica e $34,2 \%$ ao de tubetes. É pertinente observar que a agulha utilizada no processo de anestesia odontológica (Resíduo Classe A/ Resíduos Infectantes Tipo A4 / Perfurante - Cortante) deve ser descartada em lixo hospitalar (CONAMA, 2001). Como os ciclos de coleta são variáveis de acordo com cada região do país, ocorre, em muitos casos, que a mesma é simplesmente descartada para o lixo comum. O tubete utilizado também é classificado como lixo hospitalar, devido à possibilidade da ocorrência de um refluxo de sangue durante a anestesia. Desta forma, são descartados pouco mais de $4800 \mathrm{~kg}$ de agulhas/embalagens e tubetes a cada milhão de anestesias aplicadas. Este valor, no ano de 2002, foi equivalente a 840 $\mathrm{kg}$ de resíduos.

\section{Consumo de energia elétrica}

O maior consumo de energia elétrica no ciclo de vida da seringa odontológica se dá nos consultórios odontológicos devido a utilização de autoclaves no processo de esterilização. Um fator importante é a introdução no mercado de novos modelos de autoclaves que consomem, em média, $20 \%$ menos energia elétrica.

\section{Com a mudança proposta para a manufatura, de resíduos sólidos no ciclo de vida corresponde a $6,50 \% \mathrm{~kg} / \mathrm{kg}$ do total.}

Deve-se ressaltar que a solução encontrada para minimizar o resíduo sólido na manufatura possibilita ainda uma redução no consumo de energia elétrica devido ao menor tempo de usinagem. Os dados obtidos após a introdução de melhorias - tubos substituindo vergalhões - para a usinagem do tubo e do garfo mostram que o consumo de energia elétrica foi reduzido de $190,76 \mathrm{kWh}$ para 103,01 $\mathrm{kWh}$ e de $135,54 \mathrm{kWh}$ para $107,08 \mathrm{kWh}$, respectivamente, o que acarretou uma redução total de 19,95\% em relação à produção de 2002. A Figura 6 mostra uma estimativa dos resultados considerando uma diminuição proporcional de $19 \%$ nas etapas de mineração e beneficiamento. Na etapa de uso, simulou-se a adoção de autoclaves com uma redução de consumo de $20 \%$.

\section{COMENTÁRIOS FINAIS}

O principal resultado do ICV da seringa odontológica para anestesia foi o conhecimento detalhado de cada etapa de produção, que permitiu identificar possibilidades de melhoria e propor modificações em parte do ciclo, promovendo desde a substituição de matérias-primas até a promoção de descarte mais eficiente.

Com a substituição dos vergalhões de latão por tubos do mesmo material, seria possível não só reduzir a geração de resíduos sólidos, mas também diminuir o consumo de energia elétrica de forma significativa. É importante destacar que, apesar da contribuição da manufatura, tanto na geração de resíduos como no consumo de energia elétrica, ser pequena em relação aos totais referentes ao ciclo completo, uma modificação nesta etapa pode resultar em 
benefícios consideráveis, que podem ocorrer tanto nas etapas anteriores (redução da necessidade de mineração e beneficiamento) como nas posteriores (redução no descarte de embalagens).

A Figura 7 mostra que, com a mudança proposta para o processo de manufatura, a redução total de resíduos sólidos no ciclo de vida da seringa odontológica seria equivalente à eliminação dos resíduos do beneficiamento, que correspondem a aproximadamente $6,50 \%$ do total. No caso da energia elétrica as mudanças propostas, apesar de representarem $20 \%$ do consumo da manufatura, não são suficientes para diminuir de forma significativa o total de energia elétrica consumido no ciclo de vida da seringa odontológica.

Nota-se, tanto no caso do descarte de resíduos sólidos como no caso do consumo de energia elétrica, que as maiores quantidades estão associadas ao uso do conjunto (seringa, agulha e tubete anestésico). Na hipótese de se usar autoclaves com consumo $20 \%$ menor, a redução no consumo de energia elétrica seria equivalente à energia consumida para a produção de polietileno, que corresponde a $20 \%$ do consumo total de energia elétrica no ciclo de vida da seringa. $\mathrm{O}$ descarte das embalagens de polietileno na etapa de uso corresponde a $45 \%$ do resíduo sólido desta etapa e a $32 \%$ do total do ciclo de vida da seringa odontológica. Desta forma, ações visando a desmaterialização das embalagens das agulhas para anestesia e a modernização das autoclaves utilizadas pelos dentistas reduziriam significativamente o potencial de impacto ambiental associado à aplicação de anestesias. É importante ressaltar que estas ações estão fora dos domínios da manufatura de seringas e que seria necessária a colaboração dos responsáveis por cada etapa do ciclo para que as modificações nos produtos e nos processos pudessem resultar na máxima redução do descarte de resíduos sólidos e do consumo de energia elétrica.

Figura 5: Distribuição dos resíduos sólidos no ciclo de vida da seringa odontológica (UF = $10^{6}$ anestesias aplicadas] considerando-se as quantidades de resíduos do processo descrito (em cinza) e após as mudanças propostas para a manufatura (em preto).

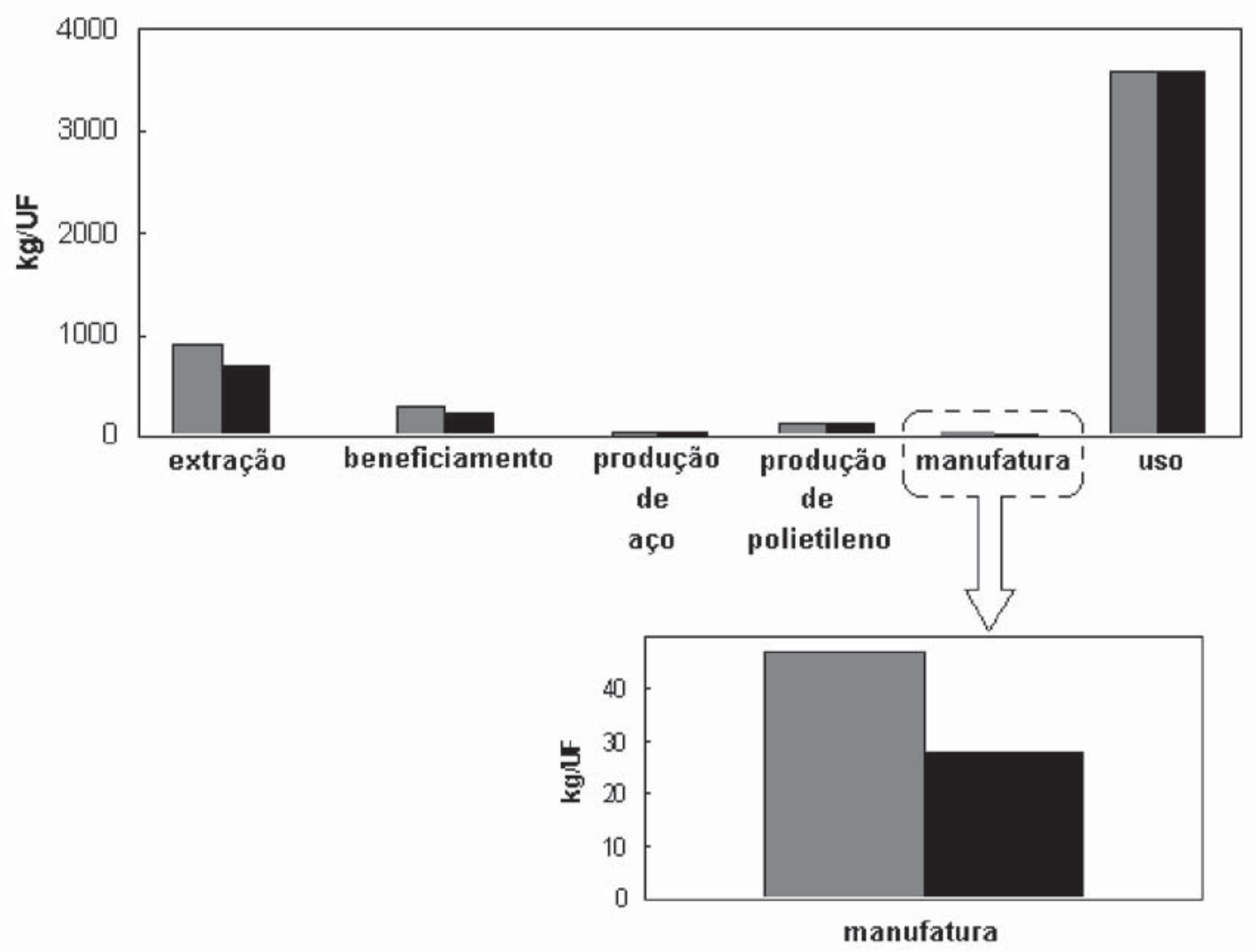


Figura 6: Consumo de energia elétrica no ciclo de vida da seringa odontológica (UF $=10^{6}$ anestesias aplicadas) considerando-se as quantidades de resíduos do processo descrito (em cinza) e após as mudanças propostas para a manufatura (em preto).

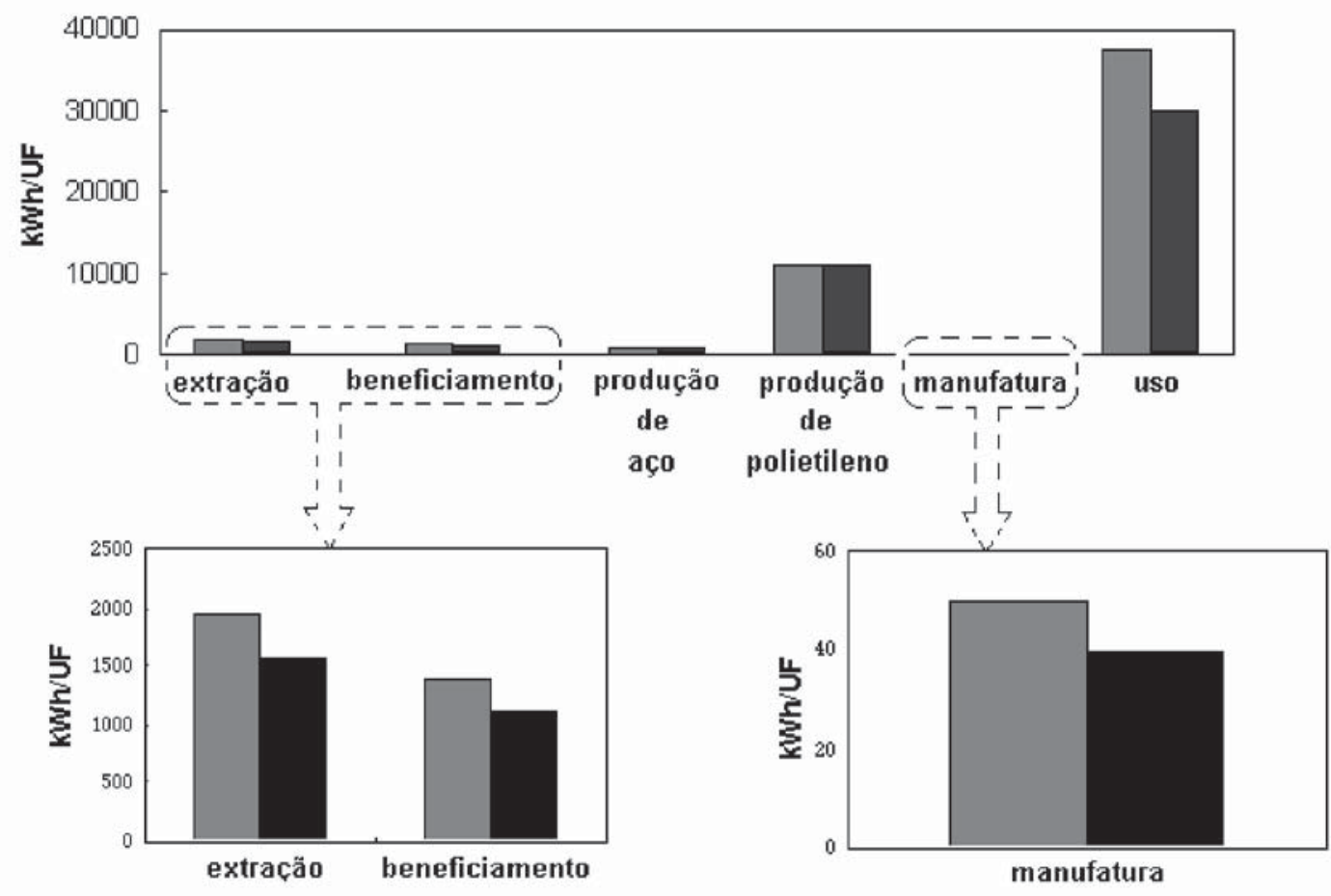

Figura 7: Quantidades totais reduzidas (em preto) de resíduos sólidos e energia elétrica no ciclo de vida da seringa odontológica (UF $=10^{6}$ anestesias aplicadas) comparadas com as quantidades de resíduos sólidos e energia elétrica antes das mudanças propostas para a manufatura (em cinza).
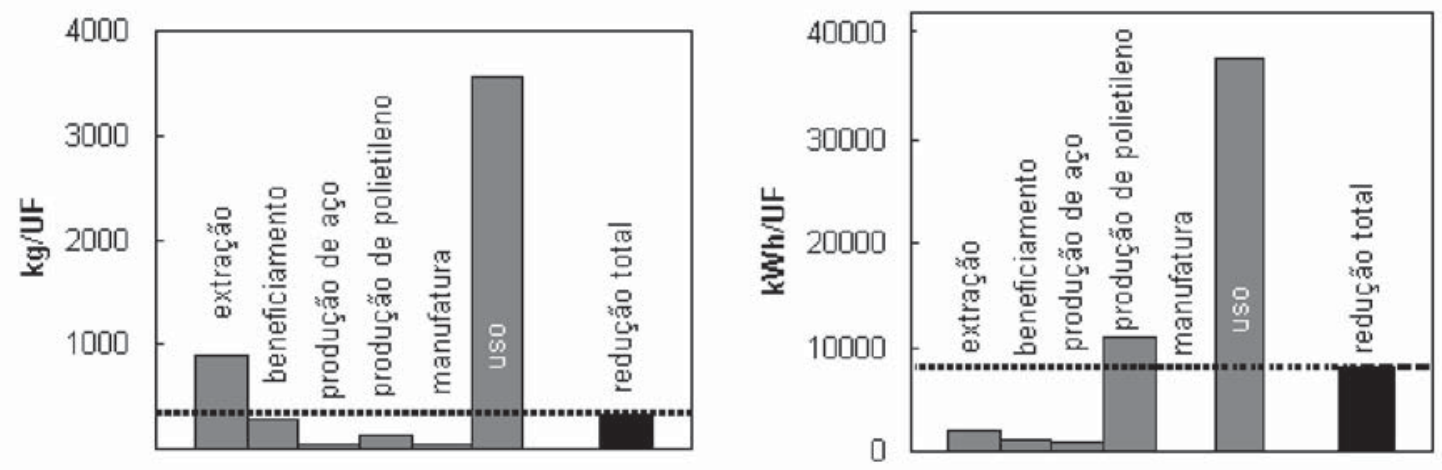


\section{Artigo recebido em 06/02/2007 \\ Aprovado para publicação em 17/08/2007}

\section{- Agradecimentos}

Os autores agradecem à Metalúrgica Farbe Ltda. pelo fornecimento dos dados necessários para a execução deste estudo.

\section{- Referências}

AHBE, S.; BRAUNSCHWEIG, A.; MIILLERWENK, R. Methodik fiir Okobilanzen auf der Basis Skologischer Optimierung, Schriftenreihe Umwelt Nr. 133. Bundesamt fur Umwelt, Wald und Landschaft. BUWAL, Berna, 1990.

AHMADI, A.; WILLIAMSON, B. H.; THEIS, T. L.; POWERS S. E. Life-cycle inventory of toner produced for xerographic processes, Journal of Cleaner Production, 11, p. $573-582,2003$.

ALTING, L.; LEGARTH, J. B. Life cycle engineering and design. Annals of the CIRP, v. 44, n. 2, 1995.

ANDERSSON, K.; OHLSSON, T.; OLSSON, P. Screening life cycle assessment (LCA) of tomato ketchup: a case study. Journal of Cleaner Production, 6, p. 277-288, 1998.

ASSOCIAÇÃO BRASILEIRA DE NORMAS TÉCNICAS (1996). NBR ISO 14001 Sistema de Gestão Ambiental - Especificação e diretrizes para uso. Brasil: ABNT. $22 \mathrm{p}$.

ASSOCIAÇ̃̃O BRASILEIRA DE NORMAS TÉCNICAS (2001). NBR ISO 14040 Gestão Ambiental - Avaliação do ciclo de vida Princípios e estrutura. Brasil: ABNT. Novembro. $10 \mathrm{p}$.

ASSOCIAÇÃO BRASILEIRA DE NORMAS TÉCNICAS (1998) NBR ISO 14041: Gestão ambiental: Avaliação do Ciclo de Vida: definições de escopo e análise do inventário. Rio de Janeiro.

ASSOCIAÇÃO BRASILEIRA DE NORMAS TÉCNICAS (2004) NBR ISO 14042: Gestão ambiental: Avaliação do Ciclo de Vida: avaliação do impacto do ciclo de vida. Rio de Janeiro.

ASSOCIAC̄̃O BRASILEIRA DE NORMAS TÉCNICAS (2005) NBR ISO 14043: Gestão ambiental: Avaliação do Ciclo de Vida: interpretação do ciclo de vida. Rio de Janeiro.
AYRES, R. U.; AYRES, L. W.; RÅDE, I. The Life Cycle of Copper, its Co-Products and By-Products, report commissioned by the MMSD (Mining, Minerals and Sustainable Development) project of IIED (International Institute for Environment and Development), World Business Council for Sustainable Development (WBCSD), 2002.

AZAPAGIC, A.; CLIFT, R. The application of life cycle assessment to proces optimization. Computers and Chemical Engineering, 23, p. 1509-1526, 1999.

BOUSTEAD, I. Ecoprofiles of the European plastics industry, POLYOLEFINS, a report for the Technical and Environmental Centre of the Association of Plastics Manufacturers in Europe (APME). APME, Brussels, 2003.

BURGESS, A. A., BRENNAN, D. J., Application of life cycle assessment to chemical processes. Chemical Engineering Science, 56, p. 2589-2604, 2001.

CEDERBERG, C.; MATTSSON, B. Life cycle assessment of milk production - a comparison of conventional and organic farming, Journal of Cleaner Production, 8 , p. 49-60, 2000.

CFO - Conselho Federal de Odontologia, http://www.cfo.org.br/index.htm. acesso em 2003.

CONAMA. Resolução no 283 de 12 de julho de 2001.

DE SMET, B.; WHITE, P. R.; OWENS, J. W. Integrating Life Cycle Assessment within an Overall Framework for Environmental Management. Em CURRAN, M. A., Ed. Environmental Life Cycle Assessment, McGraw-Hill Companies, Nova Iorque, 1996.
FINNVEDEN, G. Methodological aspects of life cycle assessment of integrated solid waste management systems. Resources, Conservation and Recycling, 26, p. $173-187,1999$.

FINNVEDEN, G.; EKVALL, T. Life-cycle assessment as a decision-support tool the case of recycling versus incineration of paper. Resources, Conservation and Recycling, 24, p. 235-256, 1998.

FURUHOLT, E. Life cycle assessment of gasoline and diesel. Resources, Conservation and Recycling, 141, p. 251-263, 1995.

GEORGAKELLOS, D. A. Evaluation of life cycle inventory results using critical volume aggregation and polygon-based interpretation. Journal of Cleaner Production, 13, p. 567-582, 2005.

IISI, Life Cycle Inventory Data for Steel Products, IISI - International Iron and Steel Institute, Bruxelas, 2000.

ISO, International Standardization Organization. Environmental Management-Life Cycle AssessmentPrinciples and Framework. International standard ISO 14040, 1. ed., Genebra: ISO, 1997.

ISO/TR14047, International Standardization Organization. Environmental Management - Life Cycle Impact Assessment - Examples of application of ISO 14042, Genebra: ISO, 2003.

NICOLETTI, G. M.; NOTARNICOLA, B.; TASSIELLI, G. Comparative Life Cycle Assessment of flooring materials: ceramic versus marble tiles. Journal of Cleaner Production, 10, p. 283-296, 2002.

PAPASAVVA S,; KIA, S.; CLAYA, J.; GUNTHER R. Characterization of automotive paints: an environmental impact analysis. Progress in Organic Coatings, 43, p. 193-206, 2001.
REPORT OF ENERGY AND ENVIRONMENTAL PROFILE OF THE U.S Mining Industry, Office of Industria Technologies, Office of Energy Efficiency and Renewable Energy, cap. 5, 2001a, $<$ http://www.eere.energy.gov/industry/ mining/pdfs/miningbro05.pdf $>$. Acesso em 2003.

REPORT OF ENERGY AND ENVIRONMENTAL PROFILE OF THE U.S. Mining Industry, Office of Industrial Technologies, Office of Energy Efficiency and Renewable Energy, cap. 6, 2001b, http://www.eere.energy.gov/industry/ mining/pdfs/miningbro06.pdf. Acesso em 2003.

RIBEIRO, C. M.; ALMEIDA, C. M. V. B.; GIANNETTI, B. F Avaliação de ciclo de vida (ACV): uma ferramenta importante da ecologia industrial, Revista de Graduação da Engenharia Química, 11, p. 13-23, 2003. Acesso eletrônico: <http://www.hottopos.com/regeq12 index.htm $>$.

RYDH, C. J.; SUN, M. Life cycle inventory data for materials grouped according to environmental and material properties. Journal of Cleaner Production, 13, p. 1258-1268, 2005

SEYLER, C.; HOFSTETTER, T. B. HUNGERBUHLER, K. Life cycle inventory for thermal treatment of waste solvent from chemical industry: a multi-input allocation model. Journal of Cleaner Production, 13, p. 1211-1224, 2005.

SILVA, G. A.; KULAY, L. A. Environmental performance comparison of wet and thermal routes for phosphate fertilizer production using LCA A Brazilian experience. Journal of cleaner production. Inglaterra, 13, p. 1219-1223, 2005.

SILVA, G. A.; BORGES, F. J. Life-cycle inventory of polyvinyl chloride manufacture in Brazil. SETA C Globe, Pensacola, 5, p. 60-61, 2004. 


\section{Referências}

SILVA, G. A.; KULAY, L. A. Application of life cycle assessment to the LCA case studies single superphosphate production. The International Journal of Life Cycle Assessment. Landsberg, 8, p. 209-214, 2003.

SOMBEKKE, H. D. M.; VOORHOEVE, D K.; HIEMSTRA P. Environmental impact assessment of groundwater treatment with nanofiltration. Desalination, 113 p. 293-296, 1997.

SEPPÄLA, J.; MELANEN, M.; JOUTTIJARVI, T., KAUPPI, L., LEIKOLA, N., Forest industry and the environment: a life cycle assessment study from Finland. Resources, Conservation and Recycling, 23, p. $87-105,1998$
SEPPÄLA, J.; KOSKELA, S.; MELANEN, M.; PALPERI, M. The Finnish metals industry and the environment. Resources, Conservation and Recycling, 35, p. 61-76, 2002.

STEEN, B.; RYDING, S. O. The EPS enviro-accounting method. IVL Report B1080, Swedish Environmental Research Institute, Gothenburg, Suécia, 1992.
TILLMAN, A. M. Environmental Impact Assessment Review, 20, p. 113-123, 2000

VERSCHOOR, A. H.; REIJNDERS, L. The use of life cycle methods by seven major companies. Journal of Cleaner Production, 7, p. 375-382, 1999.

- Sobre os autores

\section{Biagio F. Giannetti}

Universidade Paulista - UNIP

Prof. Titular do curso de Pós-graduação em Engenharia de Produção

Área de Concentração: Produção e Meio Ambiente.

Linha de Pesquisa: Produção Mais Limpa e Ecologia Industrial

E-mail: biafgian@unip.br

\section{Cecília M. Villas Bôas de Almeida}

Universidade Paulista - UNIP

Profa. Titular do curso de Pós-graduação em Engenharia de Produção

Área de Concentração: Produção e Meio Ambiente.

Linha de Pesquisa: Produção Mais Limpa e Ecologia Industrial

E-mail: cmvbag@unip.br

\section{Silvia H. Bonilla}

Universidade Paulista - UNIP

Profa. Titular do curso de Pós-graduação em Engenharia de Produção Área de Concentração: Produção e Meio Ambiente.

Linha de Pesquisa: Produção Mais Limpa e Ecologia Industrial

E-mail: bonilla@unip.br

\section{Celso Munhoz Ribeiro}

Universidade Paulista - UNIP

Mestre em Engenharia de Produção - Produção e Meio Ambiente

E-mail: profcelsoribeiro@unip.br

End.: Rua Dr. Bacelar, 1212 - São Paulo - SP - CEP 04026-002

Tel.: (11) 5586-4127 